\title{
Shortcomings in the Work of Food Safety Management Teams in My Country and Optimization Strategies
}

\author{
Yong Huang $^{* 1}$, Qingqing Liu ${ }^{2}$ \\ ${ }^{1}$ SEGi University, Patalin Jaya, Malaysia. E-mail: yong0017@ hotmail.com \\ ${ }^{2}$ University of Hull, Hull HU6 7RX, United Kingdom.
}

\begin{abstract}
Relevant surveys show that in recent years, with the improvement and optimization of the level of economic development, the public's attention to catering has been increasing, which has effectively promoted the development and implementation of food safety management in my country. In this process, a large number of researchers have conducted in-depth analysis and exploration of related work based on the current development of my country's food safety management team, aiming to further improve and optimize the comprehensive level of food safety management in order to work for my country's food safety. The improvement of overall quality provides a new impetus. Based on a large amount of data, this paper analyzes and discusses the shortcomings of my country's food safety management at this stage. At the same time, the researchers put forward corresponding optimization strategies based on their own work experience, aiming to further realize the comprehensive quality of the food safety management team. The improvement and optimization of food safety in China will then provide impetus for the development of my country's food safety work.
\end{abstract}

Keywords: Food Safety; Management Team; Daily Work; Main Issues; Optimization Strategy

Researchers pointed out that in daily work, the rational development and implementation of food management team work is conducive to the realization of a reasonable guarantee of food hygiene and health in my country, and it has good promotion significance and value for the maintenance of people's dietary health. On this issue, in recent years, with the emergence of a large number of food safety incidents, the public has shown a high degree of concern about the work of the food safety management team. Based on this, the relevant departments should actively conduct a systematic analysis and reasonable reflection on related work content, so as to effectively respond to the concerns of all sectors of society, and then lay a solid foundation and guarantee for the improvement and optimization of the comprehensive capabilities of my country's food safety management team. On this issue, in order to reasonably achieve effective solutions to related issues, relevant departments should reflect on the current situation of their own work in light of the objective situation, and at the same time formulate corresponding optimization countermeasures.

\section{The important value of food safety management team work}

As the main organizer and implementer of food safety work in my country, the work of food safety man-

Copyright (C) 2021 Yong Huang et al.

doi: $10.18686 /$ fsr.v3i1.1366

This is an open-access article distributed under the terms of the Creative Commons Attribution Non-Commercial License

(http://creativecommons.org/licenses/by-nc/4.0/), which permits unrestricted non-commercial use, distribution, and reproduction in any medium,

provided the original work is properly cited. 
agement teams is often closely related to the food safety of the people. Based on this, in recent years, most staff have conducted in-depth analysis and research on the safety of dietary structure in combination with related content, aiming to further improve and optimize the overall level of management work, so as to effectively achieve diet safety in the daily lives of the people of our country. The reasonable guarantee and effective maintenance of the health of the people lay a solid foundation for the effective maintenance of the health of the people. At the same time, the researchers pointed out that through the implementation of the food safety management team, it is conducive to the systematic identification of additives and harmful substances in food, and has a good guiding role in the timely resolution of potential problems, which is conducive to the realization of my country. The further improvement of the food safety supervision system has laid a solid foundation and guarantee for Chinese food to better enter the world market.

\section{The shortcomings in the daily work of my country's food safety management team}

\subsection{Insufficient professional competence of staff is not conducive to the smooth devel- opment of management}

Generally speaking, in the process of food safety management team work, the professional ability and professionalism of the staff often have a serious impact on the work level. However, at present, the professional ability of the staff of the food safety management team in my country is relatively low. Based on this, in the process of daily work, some staff often find it difficult to reasonably realize the systematic analysis and exploration of related work. The improvement and optimization of the overall quality of work has caused extremely adverse effects. In response to this problem, the researchers pointed out that at this stage, some food safety management departments have relatively low barriers to entry for their staff. Therefore, in the food safety management team, the professional capabilities of some staff are relatively weak. It is often difficult to reasonably realize the systematic analysis and exploration of related work, which limits the improvement of the comprehensive ability of management work, and has an extremely nega- tive impact on the optimization of management work quality. On the other hand, a large amount of data shows that due to the lack of management skills, it is often difficult for staff to effectively realize timely detection and reasonable resolution of potential problems while carrying out food safety management, which is extremely disadvantageous for the reasonable guarantee of the comprehensive quality of food management work impact.

\subsection{The management work system is not perfect, which is not conducive to the rea- sonable implementation of management work}

In the process of food safety management work, some management teams have not yet effectively established and improved the work system. Based on this, in the process of daily work, it is often difficult for relevant staff to reasonably realize the detailed development of specific work, which has extremely adverse effects on the improvement and optimization of work management level, and thus restricts the comprehensive food safety management work in my country. On the other hand, due to the lack of systems, related management work is often difficult to be standardized, which is not conducive to the improvement of the comprehensive ability of management work, and has caused serious impact and obstacles to the optimization of the comprehensive level of management work. In addition, due to the lack of management work systems, it is difficult for staff to realize timely detection and reasonable resolution of potential work problems while performing daily work, which has an adverse impact on the systematic analysis of work content.

\subsection{Low management skills are not condu- cive to effective guarantee of work efficiency}

A large number of survey data show that at this stage, in the process of my country's food safety management work, the level of inspection technology applied by some management teams is relatively weak. Based on this, it is often difficult to achieve relevant results in the process of inspection work. The systematic analysis and effective understanding of the problem, in turn, have extremely adverse effects on the reasonable solution and effective regulation of potential problems. Specifically, due to the existence of this factor, the food 
inspection work efficiency is low, and the inspection work accuracy is relatively weak, which is not conducive to the improvement of the overall quality of the inspection work, and has a negative impact on the optimization of the inspection effect.

\section{Strategies to improve the com- prehensive level of my country's food safety management team}

\subsection{Strengthen the learning of professional knowledge and enhance the comprehensive ability of the management team}

For food safety management staff, in order to further improve and optimize the comprehensive level of related work, they should actively study and explore related knowledge in their daily work, so as to further realize a full understanding of food inspection skills. Effective control to ensure that food inspection can be carried out and implemented effectively according to relevant content in daily work, so as to lay a solid foundation and guarantee for the development of comprehensive quality of food inspection. On the other hand, in this process, in order to effectively achieve the improvement of the comprehensive strength of the staff, the relevant departments should guide the relevant staff to discuss and analyze related issues on a regular basis, so as to achieve effective solutions to specific problems and improve the comprehensive capabilities of the staff to cultivate and lay a solid foundation. Practice has shown that through the development and implementation of the discussion link, relevant departments can guide the staff to further realize the cultivation and mobilization of thinking activity during the discussion process, so as to help them fully recognize professional knowledge, and provide good for the orderly development and implementation of follow-up work under ideological conditions.

\subsection{Improve management work system to ensure effective implementation of manage- ment work}

In the process of food safety management work, in order to further realize the effective implementation and practice of related work, the management department should actively do a systematic analysis and effective exploration of related work, so as to effectively formu- late the management work system based on the actual situation. In order to achieve reasonable satisfaction of the comprehensive needs of management work, and provide impetus for the improvement and optimization of the comprehensive quality of management work. On this issue, a large amount of data shows that through reasonable analysis of relevant content, management staff can further improve and optimize the quality of management work, thereby ensuring the effective practice of specific management work. In addition, through the formulation of relevant systems, staff can better analyze their own work conditions, which has a good guiding role in the cultivation of staff's comprehensive ability.

\subsection{Introduce advanced management tech- nology to promote the steady improvement of management efficiency}

Researchers pointed out that in order to effectively change the problem of low efficiency of food safety management in my country, relevant work teams should actively conduct reasonable analysis and effective exploration of management work, so as to actively do a good job in the development and introduction of advanced management technology in order to achieve my country. The improvement of the efficiency of product research and development management has laid a solid foundation and guarantee for the optimization of the overall quality of management work. In response to this problem, related studies have shown that through the introduction of advanced technology, the management department can better realize the analysis of management work, thereby ensuring the improvement of management efficiency. On the other hand, based on advanced technology, it is possible to better guarantee the inspection results of life insurance products, and it has a good role in promoting the improvement of inspection accuracy and the protection of food safety for the people. For example, through the application of information technology, the establishment and improvement of the database can be effectively realized, so as to realize the traceability of the inspected products in a timely manner, which has a good value in promoting the overall quality of food supervision.

\section{Conclusion}

As the main organizer and implementer of food 
management work, the work level of the food safety management team is often related to the food safety of the people. On this issue, through analyzing a large amount of data, the researchers pointed out that at this stage, the comprehensive ability of my country's food safety management team is relatively weak. Based on this, in order to further improve and optimize management efficiency, relevant staff actively do a good job in the analysis and exploration of related content, so as to optimize the self-discipline and improvement of the optimization strategy based on its own work situation, so as to effectively realize the improvement of the comprehensive level of food safety management work, and provide a new impetus for the further optimization of the safety of my country's food industry.

\section{References}

1. Lin X. Status quo and problem analysis of cost management in urban rail transit enterprises (in Chinese). Accounting Learning 2019; (33): 161, 163.
2. Chen $X$. The present situation, problems and solutions of PPP model of urban rail transit in China. Journal of Hunan Finance and Economics University 2019 ; 35(4): 65-72.

3. Chen T. Analysis on the status quo and development measures of urban rail transit operation and management in my country (in Chinese). Create Living 2019; (7): 181-182.

4. Ji W. Strategic guidance and quality upgrade of city comprehensive traffic development. Shanghai Urban Management 2016; 25(1): 62-65.

5. Liu T. On the teaching status of urban rail transit operation management major in higher vocational colleges (in Chinese). Modern Economic Information 2019; (4): 436.

6. Xu Z. Safety management status and management ability improvement of rail transit construction supervision units (in Chinese). Technology and Industry Across the Straits 2017; (5): 75-76.

7. Dong X. Comparative analysis of the causes of traffic congestion in large cities and countermeasures: A comparative analysis of the current situation of the traffic systems in Wuhan and Hangzhou as an example (in Chinese). Cultural Geography 2016; (6): $30-31$. 